\title{
Surgical Outcome of Fusion in Recurrent Lumbar Disc Herniation
}

\author{
Ahmed Taha* (), Mohamed Youssef \\ Neurosurgery Department, Faculty of Medicine, Al-Azhar University, Damietta, Egypt \\ Email:^ahmdth231@gmail.com,youssef200877@yahoo.com
}

How to cite this paper: Taha, A. and Youssef, M. (2020) Surgical Outcome of Fusion in Recurrent Lumbar Disc Herniation. Open Journal of Modern Neurosurgery, 10, 157-166.

https://doi.org/10.4236/ojmn.2020.101016

Received: March 1, 2019

Accepted: March 25, 2019

Published: December 31, 2019

Copyright $\odot 2020$ by author(s) and Scientific Research Publishing Inc. This work is licensed under the Creative Commons Attribution International License (CC BY 4.0).

http://creativecommons.org/licenses/by/4.0/ (c) (i) Open Access

\begin{abstract}
Background Data: Recurrent lumbar disc herniation means re-herniation of disc on the same site and at the same level where a previous discectomy had been performed: recurrent lumbar disc herniation occurs in $7 \%$ to $24 \%$ of patient underwent discectomy. Tow mean surgical options after reherniated lumbar disc are revision discectomy alone or revision discectomy with fusion discectomy alone in recurrent lumbar disc herniation may not be an efficient treatment without fusion. Objective: To evaluate the efficacy and safety of revision discectomy with fusion in management of recurrent lumbar disc. Study Design: Retrospective study reviewed all patient underwent revision discectomy with fusion, they were 40 patients operated for recurrent lumbar disc from September 2014 to April 2018 in Al-Azhar University Hospital Damietta. Pre- and post-operative data collection and analysis of the outcome were completed based on the "Japanese Orthopedic Association score" (JOAs), and radiographic follow-up. Patients and Methods: 40 patients (30 male and 10 female) underwent revision discectomy with fusion as surgical management for reherniated lumbar disc from September 2014 to April 2018. All patients presented with low back pain and radicular pain with mean duration of 18 months. The patients were investigated by standard plain X-ray CT SCAN and MRI of the lumbar spine. All patients had a discectomy and postero-lateral fusion in revision surgery. These patients followed post operatively clinically for improving pain and neural function, and radiologically for disc removal stability and fusion. Results: The age ranged from 30 to 60 years, mean age was 45 years, male to female ratio 3:1. Follow-up ranged from 18 - 30 months with a mean follow-up 24 months. 30 patients had an excellent outcome, 6 patients had a good outcome, 2 patients had a fair outcome, and 2 patients had a poor outcome. Conclusion: Recurrent lumbar disc herniation occurs in $7 \%$ to $24 \%$ of patient underwent discectomy. Revision surgery when indicated can be done by various techniques. Revision discectomy with fusion for reherniated lumbar disc is effective and safe with confident results.
\end{abstract}




\section{Keywords}

Reherniated Lumbar Disc, Postero-Lateral Fusion, Revision Discectomy

\section{Introduction}

Recurrent disc herniation is defined as disc herniation seen at the same level after a painless period of at least 6 months following the first surgery. Recurrent herniation may be on the same or opposite side [1] [2].

Risk of unfavourable outcomes like obesity, diabetes, vibration workers, drivers and psychological factors should be taken into consideration before surgery for the first instance which increases the incidence of recurrence [3].

The reoperation rate following the initial lumbar discectomy ranges between $4 \%$ and $18 \%[4]$.

The optimal treatment of recurrent disc herniation is still controversial. Some surgeons choose simple discectomy again, while some surgeons advocate fusion surgery. Because recurrent disectomy (ipsilateral/contralateral) requires more disc and posterior spinal component removal (lamina and/or facet joint), recurrent discectomy will increase the likelihood of segmental instability and due to scar tissue; dural tear, and nerve injuries may be greater at simple re-discectomies, some surgeons suggest fusion surgery at first recurrent, regardless of whether instability [5].

The aim of this study was to evaluate the efficacy and safety of fusion in reherniated lumbar disc surgery.

\section{Patients and Methods}

This retrospective study was carried out on 40 patients 30 male and 10 female age ranged from 30 to 60 years with mean age 45 years who underwent revision discectomy with fusion for reherniated lumbar disc from September 2014 to April 2018 in Neurosurgery Department Al-Azhar University Hospital New Damietta Egypt.

Inclusion criteria:

1) At least 6 months of pain relief after previous disc surgery.

2) The presence of recurrent disc herniation and radicular pain not respond to conservative treatment.

Exclusion criteria:

1) Those with cauda equine syndromes.

2) Patients with spondylolithesis.

3) Reoperation in the early postoperative period for infections.

The study was approved by the local ethical committee and informed consent was obtained from participating patients. Data collection was extracted from hospital records (patient admission sheet medical records imaging studies operative and postoperative details and progressive notes). 


\section{Preoperative Assessment}

All patients were subjected to careful history taking general and neurological examination. All patients had pre operative lumbo-sacral X-ray A-P and dynamic views flexion extension and oblique and MRI examination (Figures 1-3).

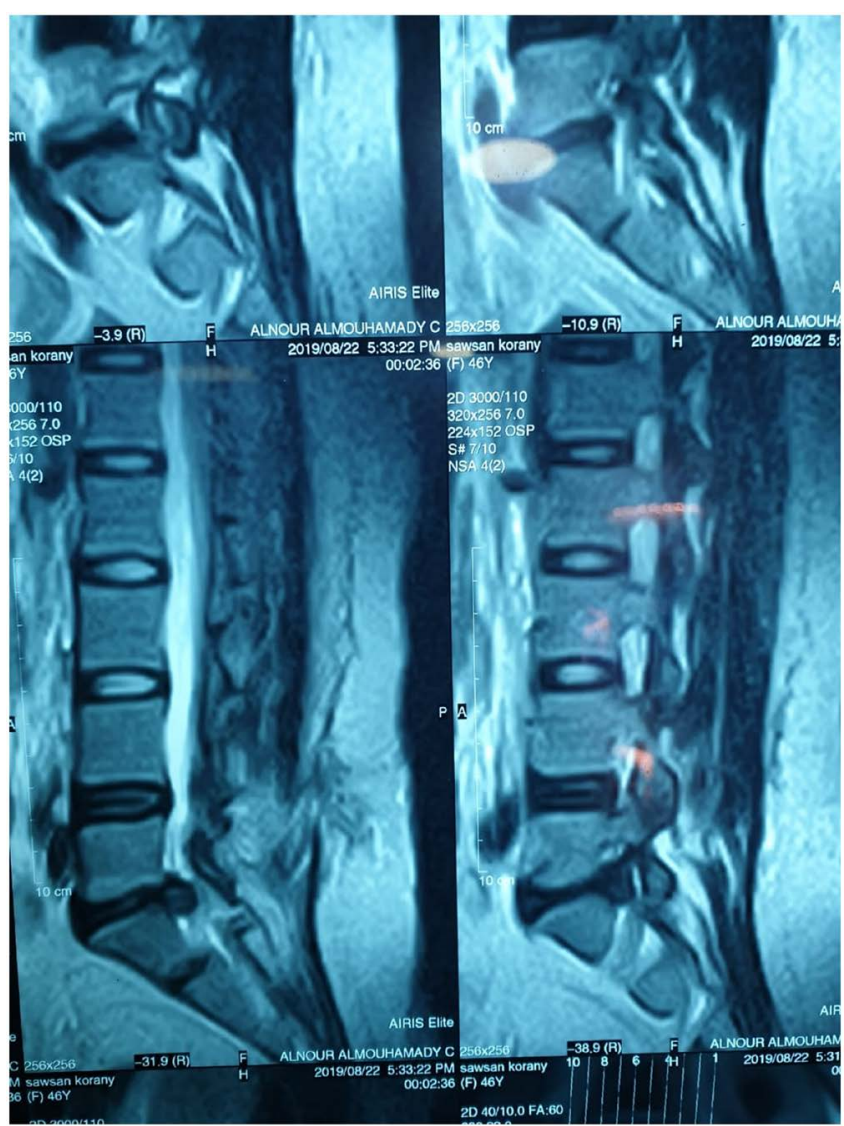

Figure 1. MRI lumbo sacral spine sagittal view show recurrent disc L5 S1.

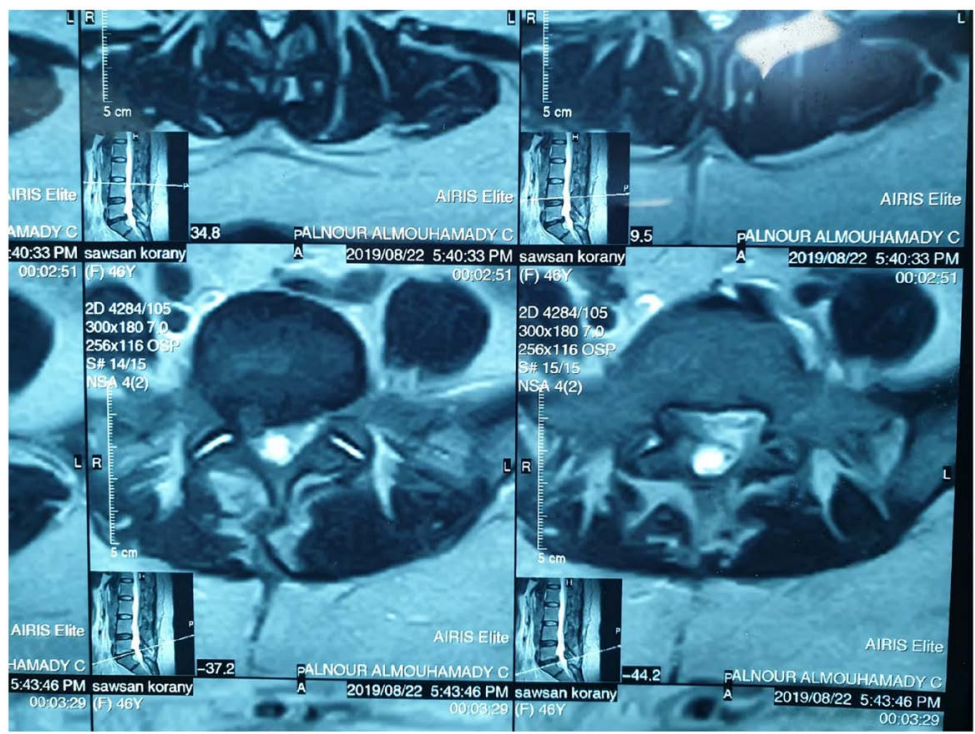

Figure 2. MRI lumbo sacral spine axial view show recurrent disc L5 S1. 


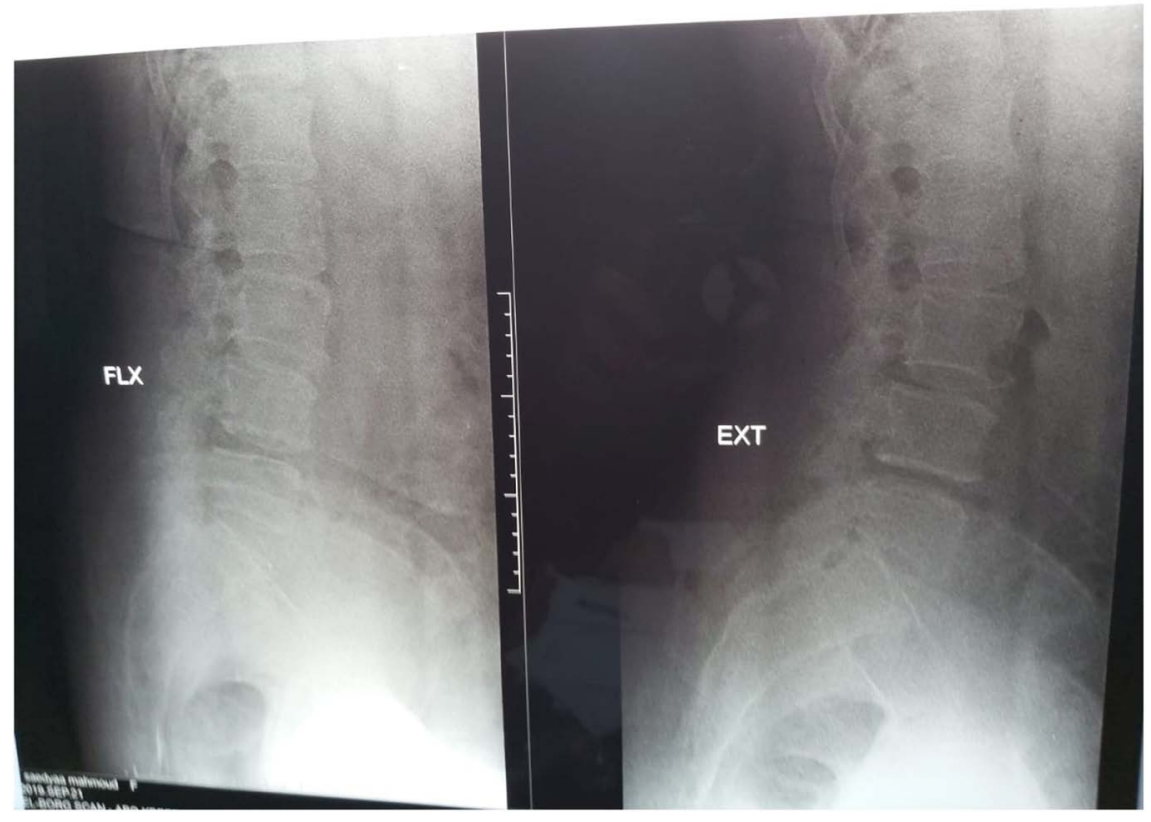

Figure 3. Preoperative plain X-ray dynamic view.

Full medical history taking all patients asked about allergies, chronic diseases as diabetes, hypertension, heart diseases, and ask about smoking, previous surgery, and results of physical exams and tests may also include full data about medicines taken and personal habits, activities and work history. The results were evaluated carefully and confirmed that recurrence rate increased with heavy activities and chronic diseases as diabetes.

\section{Operative Technique}

Surgery was performed after induction of general anesthesia with the patient placed in prone position and the spine flexed. All patients were positioned prone on frame or rolls to avoid abdominal compression and hence reduce venous congestion. Skin incision was given over the previous operative site after full preparation. After muscle dissection soft tissue was cleaned from facet in a lateromedial direction. The medial edge of facet defined with curate and the plane between the dura and the medial facet was appreciated and enlarged. Medial facectetomy was completed. The nerve root identified after removing the remaining ligamentum flavum. Nerve root was retracted and the discectomy completed. Posterolateral fusion and trans-pedicular screw fixation were performed.

Closure was then done in a routine fashion after insertion of a subcutaneous suction drain (Figure 4 \& Figure 5).

Clinical symptoms were evaluated pre and post-operatively according the criteria of the "Japanese Orthopedic Associations core" (JOA) [6].

Japanese Orthopaedic Association (JOA) score assessment of the surgical treatment of low back pain (Tables 1-4). 


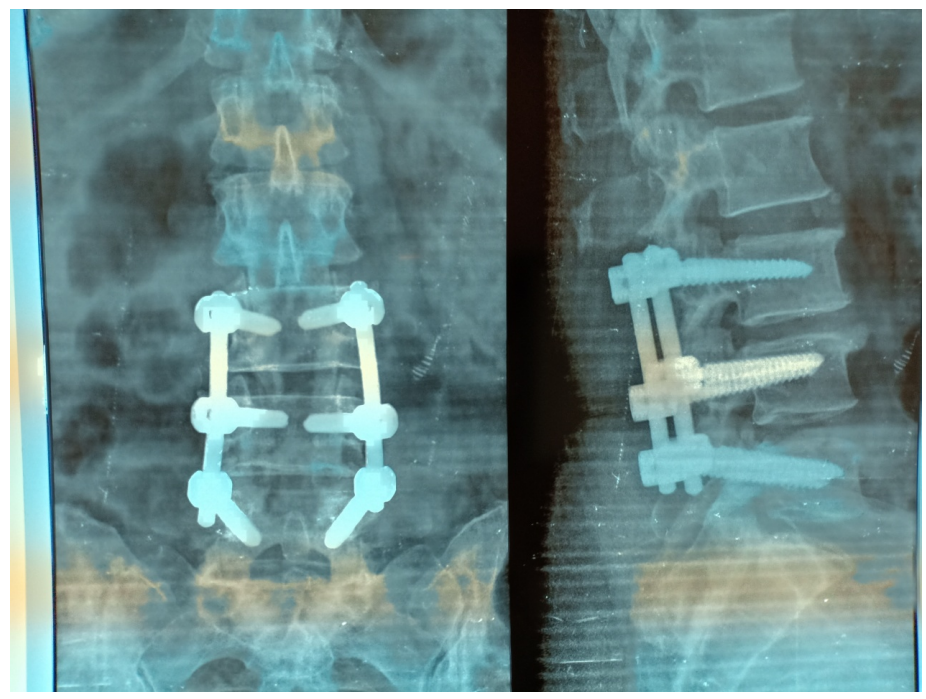

Figure 4. Postoperative plain X-ray A-P and LAT view show transpedicular fixation (screw rod system) double level L3 L4 L5.

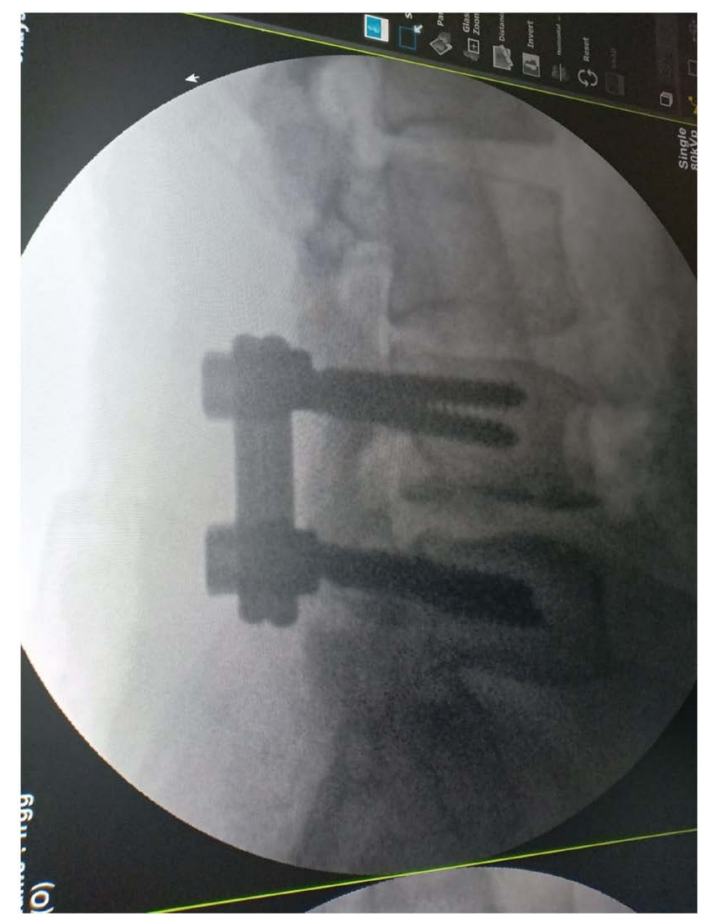

Figure 5. Postoperative plain X-ray lat view shows transpedicular fixation single level L4 L5.

Table 1. Subjective symptoms (9 points). (a) Low-back pain; (b) Leg pain and/or tingling; (c) Gait.

(a)

\begin{tabular}{lcc}
\hline 1 & None & 3 \\
2 & Occasional mild pain & 2 \\
3 & Frequent mild or occasional severe pain & 1 \\
4 & Frequent or continuous severe pain & 0 \\
\hline
\end{tabular}


(b)

\begin{tabular}{ccc}
\hline 1 & None & 3 \\
2 & Occasional slight symptoms & 2 \\
3 & Frequent slight or occasional severe symptoms & 1 \\
4 & Frequent or continuous severe symptoms & 0 \\
\hline
\end{tabular}

(c)

\begin{tabular}{llc}
\hline 1 & Normal & 3 \\
2 & Able to walk farther than $500 \mathrm{~m}$, although it results in pain, tingling, and/or muscle weakness & 2 \\
3 & Unable to walk farther than $500 \mathrm{~m}$ because of leg pain, tingling, and/or muscle weakness & 1 \\
4 & Unable to walk farther than $100 \mathrm{~m}$ because of leg pain, tingling, and/or muscle weakness & 0 \\
\hline
\end{tabular}

Table 2. Clinical signs (6 points). (a) Straight-leg raising test (including tight hamstrings); (b) Sensory disturbance; (c) Motor disturbance.

(a)

\begin{tabular}{ccc}
\hline 1 & Normal & 2 \\
2 & $30^{\circ}-70^{\circ}$ & 1 \\
3 & $<30^{\circ}$ & 0 \\
\hline & $($ b) & \\
\hline 1 & None & 2. \\
2 & Slight disturbance & 1. \\
3 & Marked disturbance & 0. \\
\hline 1 & (c) & \\
\hline 2 & Normal (grade 5) & 2 \\
3 & Slight weakness (grade 4) & 1 \\
\hline
\end{tabular}

Table 3. Restriction of activities of daily living (14 points).

\begin{tabular}{cccc}
\hline Activities of daily living & Severe & Moderate & None \\
\hline Turning over while lying & 0 & 1 & 2 \\
Standing & 0 & 1 & 2 \\
Washing & 0 & 1 & 2 \\
Leaning forward & 0 & 1 & 2 \\
Sitting (1 h) & 0 & 1 & 2 \\
Lifting or holding & 0 & 1 & 2 \\
Walking & 0 & 1 & 2 \\
\hline
\end{tabular}

Table 4. Urinary bladder function (6 points) (incontinence urinary retention).

\begin{tabular}{ccc}
\hline 1 & Normal & 0 \\
2 & Mild dysuria & -3 \\
3 & Severe dysuria & -6 \\
\hline
\end{tabular}


Surgical outcome assessed according to the recovery rate as described by $\mathrm{Hi}$ rabayashi et al. [6]

$$
\text { Recovery rate }(\%)=\frac{\text { post operative score }- \text { preoperative score }}{\text { Normal score }(29)-\text { preoperative score }} \times 100
$$

The results were classified into a four-grade scale: excellent improvement $>$ $90 \%$, good $75 \%-89 \%$, fair $50 \%-74 \%$ and poor $<49 \%$. Differences in preoperative symptoms and post-operative outcomes were statistically analyzed. The statistical significance was set at a P-value.

\section{Results}

Forty patients with recurrent lumbar disc were surgically treated 30 male 75\% and 10 female $25 \%$ the mean age at the time of surgery was 45 years range from 30 - 60 years. The recurrent time to the primary surgery ranged from $9-24$ months with a mean duration of 17 months (Table 5). The most common complaint was low back pain and radicular pain. Overall JOA score of the patients showed improvement, moving from (6.54 points) before surgery to (12.65 points) at the final follow-up. Low back pain, radicular pain ability to walk, straight leg raising, and manual muscle testing valuated by JOA score are shown in Table 6.

Surgical outcome, based on recovery rate, was excellent in 30 (75\%) patients, good in $6(15 \%)$ patients, fair in $2(5 \%)$ patients, and poor in $2(5 \%)$ patients (Table 7).

No major complications were observed. There was one case with superficial infection and they had received parental antibiotics with no need for surgical intervention. Five patients had dural tears which were repaired intra-operatively with no subsequent sequelae (Table 8).

\section{Discussion}

Reherniated lumbar is an important disease which seen commonly. The rate of recurrent disc herniation after lumbar discectomy is $5 \%$ to $15 \%$ [5]. So that, recurrent lumbar disc herniation (RLDH) is a major cause of surgical failure [7], the recurrent disc herniation is the major cause of the failed back surgery syndrome [8]. The optimal surgical approach (simple discectomy with or without fusion of the affected segment) for recurrent disc herniation remains a subject of controversy [9] [10].

Table 5. Age, sex, and duration of recurrence.

\begin{tabular}{ccc}
\hline Age (Years) & Range & $30-60$ \\
& Mean & 45 \\
Sex & Male 30 & Female 10 \\
Recurrence time & Range & 9 months -24 months \\
& Mean & 17 months \\
\hline
\end{tabular}


Table 6. Severity of clinical symptoms before and after surgery.

\begin{tabular}{ccc}
\hline & Range & Mean \\
\hline Pre-Operative JOA Score of Points & & \\
Low back pain & $0-1$ & 1 \\
Leg pain & $0-2$ & 0.6 \\
Ability to walk & $0-2$ & 1.2 \\
Straight leg raising & $0-1$ & 0.66 \\
Sensory abnormalities & $0-1$ & 1 \\
Motor weakness & $0-2$ & 1.5 \\
Post-Operative JOA Score & & 1.7 \\
Low back pain & $1-2$ & 2 \\
Leg pain & $1-2$ & 2.62 \\
Ability to walk & $0-3$ & 1.8 \\
Straight leg raising & $0-2$ & 1 \\
Sensory abnormalities & $0-1$ & 1.76 \\
Motor weakness & $0-2$ & \\
\hline
\end{tabular}

Table 7. Results assessed by JOA score.

\begin{tabular}{cc}
\hline Score & No. of Patients \\
\hline Excellent & $30(75 \%)$ \\
Good & $6(15 \%)$ \\
Fair & $2(5 \%)$ \\
Poor & $2(5 \%)$
\end{tabular}

Table 8. Reported complications.

\begin{tabular}{cc}
\hline Complication & No. of Patients \\
\hline Deep infection & 0 \\
Superficial infection & $1(2.5 \%)$ \\
Vascular injury & 0 \\
Dural tear & $5(12.5 \%)$ \\
Neurological insult & 0 \\
\hline
\end{tabular}

For this reason, its accurate management is of great importance. In the modern LDH surgery, various techniques have been identified and applied since the Love's operation was identified [11].

There is a number of case series describing outcomes after either preoperative discectomy or postoperative discectomy combined with fusion [12].

In the present study, we done revision discectomy with fusion this technique resulted in satisfactory results.

Lehmann and LaRocca treated 36 patients following previous lumbar surgery by using spinal canal exploration and spinal fusion. Solid fusion correlated 
closely with satisfactory outcomes, and the patients in the fusion group tended to have better outcomes than those with disc excision alone [13] in the present study which conducted on 40 patients with first time recurrent lumbar disc herniation after exclusion of those with cauda equine syndromes, and patients with spondylolisthesis. After a mean follow-up of 18 months, the mean postoperative recovery rate was $85 \%$ and the satisfactory rate was $90 \%$.

In the present study, the surgical outcome assessed according to JOA score, was excellent in 30 patients (75\%) good in 6 patients (15\%) fair in 2 patients (5\%) and poor in 2 patients (5\%). Tsai-Sheng et al. [10] reported in their study that, general clinical outcome, based on the JOA score, was excellent in $20(48.8 \%)$ patients, good in $13(31.7 \%)$, fair in $4(9.8 \%)$, and poor in $4(9.8 \%)$. clinical outcome was satisfactory (excellent or good) in $78.3 \%$ of patients whom received discectomy in $83.3 \%$ [10].

As regards the complications in the present study was found that 5 patients (12.5\%) had dural tear and 1 (2.5\%) patients had superficial infection. Tsai-Sheng et al., stated in their series that, there were five patients ( 3 in the non-fusion group and 2 in the fusion group) had a dural tear and one patient in the fusion group had a superficial infection [10].

Tsai-Sheng et al. [10] stated that, the optimal surgical approach (simple discectomy with or without fusion of the affected segment) for recurrent disc herniation remains a subject of controversy [10], but in the present study it was found that revision discectomy with fusion has several advantages in the surgical outcomes, specifically, lumbar fusion which minimizes segmental motion, immobilizes the spine decrease mechanical stresses across the degenerated disc space, and may reduce reherniated disc at the same level.

\section{Conclusion}

Fusion in re-herniated lumbar disc remains a controversial decision. But the surgical outcome is more satisfactory when revision discectomy is done with fusion rather than by using discectomy alone and this confirmed in our study revision discectomy with fusion in reherniated lumbar disc is a worthy choice according to our study.

\section{Conflicts of Interest}

The authors declare no conflicts of interest regarding the publication of this paper.

\section{References}

[1] Sonmez, E., Coven, I., Sahinturk, F., Yilmaz, C. and Caner, H. (2013) Unilateral Percutaneous Pedicle Screw Instrumentation with Minimally Invasive TLIF for the Treatment of Recurrent Lumbar Disk Disease: 2 Years Follow-up. Turkish Neurosurgery, 23, 372-378. https://doi.org/10.5137/1019-5149.JTN.7122-12.1

[2] Kim, K.T., Park, S.W. and Kim, Y.B. (2009) Disc Height and Segmental Motion Asrisk Factors for Recurrent Lumbar Disc Herniation. Spine, 34, 2674-2678. 
https://doi.org/10.1097/BRS.0b013e3181b4aaac

[3] Kara, B., Tulum, Z. and Acar, U. (2005) Functional Results and the Risk Factors of Reoperations after Disc Surgery. European Spine Journal, 14, 43-48. https://doi.org/10.1007/s00586-004-0695-3

[4] Fiume, D., Sherkat, S., Callovini, G.M., Parziale, G. and Gazzeri, G. (1995) Treatment of the Failed Back Surgery Syndrome Due to Lumbo-Sacral Epidural Fibrosis. Acta Neurochirurgica Supplementum, 64, 116-118.

[5] Yoo, M.W., Hyun, S.J., Kim, K.J., Jahng, T.A. and Kim, H.J. (2014) Does Obesity Make an Influence on Surgical Outcomes Following Lumbar Microdiscectomy? Korean Journal of Spine, 11, 68-73. https://doi.org/10.14245/kjs.2014.11.2.68

[6] Takeshima, T., Kabara, K., Mayata, S. and Ueda, Y. (2000) Clinical and Radiolographic Evaluation of Disc Excision for lumbar Disc Herniation with and without Posterolateral Fusion. Spine, 25, 450-456.

https://doi.org/10.1097/00007632-200002150-00010

[7] Fandiño, J., Botana, C., Viladrich, A. and Gomez-Bueno, J. (1993) Reoperation after Lumbar Disc Surgery: Results in130 Cases. Acta Neurochirurgica, 122, 102-104.

https://doi.org/10.1007/BF01446994

[8] Blamoutier, A. (2013) Surgical Discectomy for Lumbar Disc Herniation: Surgical Techniques. Orthopaedics \& Traumatology: Surgery \& Research, 99, S187-S196. https://doi.org/10.1016/j.otsr.2012.11.005

[9] Roy Camille, R., Saillant, G. and Mazel, C. (1986) Internal Fixation of the Lumbar Spine by Pedicle Screw Plating. Clinical Orthopaedics and Related Research, 203, 7-17. https://doi.org/10.1097/00003086-198602000-00003

[10] Sheng, F.T., Lai, P., Tsai, T.T., Niu, C.C. and Chen, L.H. (2005) Long Term Results of Disc Excision for Recurrent Lumbar Disc Herniation with or without Posterolateral Fusion. Spine, 30, 2830-2834.

https://doi.org/10.1097/01.brs.0000190393.15369.94

[11] Bokov, A., Isrelov, A., Skorodumov, A., Aley-Nik, A., Simonov, A. and Mlyavykh, S. (2011) An Analysis of Reasons for Failed Back Surgery Syndrome and Partial Results after Different Types of Surgical Lumbar Nerve Root Decompression. Pain Physician, 14, 545-557.

[12] Suk, K.S., Lee, H.M., Moon, S.H. and Kim, N.H. (2001) Recurrent Lumbar Disc Herniation: Results of Operative Management. Spine, 26, 672-676. https://doi.org/10.1097/00007632-200103150-00024

[13] Lehmann, T.R. and LaRocca, H.S. (1981) Repeat Lumbar Surgery. A Review of Patients with Failure from Previous Lumbar Surgery Treated by Spinal Canal Exploration and Lumbar Spinal Fusion. Spine, 6, 615-619.

https://doi.org/10.1097/00007632-198111000-00014 